\title{
Chemistry Education in Talent Training of Mineral Processing Engineering Disciplines Paper
}

\author{
Shuming Wen, Haiying Shen \\ Faculty of Land Resource Engineering, Kunming University of Science \& Technology, Kunming, China \\ Email: shmwen@126.com, shying65@126.com
}

Received 2012

\begin{abstract}
In mineral processing engineering disciplines, flotation, as a primary beneficiation method, is performed on the basis of surface chemical properties of minerals and rocks. The surface chemistry of minerals and rocks can be artificially changed to achieve minerals flotation separation. Thus chemistry plays a very important role in mineral processing engineering disciplines. Analysis shows that inorganic chemistry, organic chemistry and physical chemistry are all required courses for undergraduate students majoring in mineral processing engineering. Analytical chemistry and further quantum chemistry for advanced talent training at the graduate education level are necessary. Chemistry lays the foundation for realizing resource use in efficiency, integration and cleanliness, and plays a significant role in wastewater recycling, zero discharge and environment protection. Emphasizing chemistry education and improving chemistry expertise are an important guarantee for environment protection and sustainable development of mineral resources exploitation and utilization.
\end{abstract}

Keywords: Chemistry Education; Mineral Resources; Mineral Processing; Talent Training

\section{Introduction}

In the process of development and utilization of mineral resources, the principle of mineral separation and concentration is based on the differences in the physicochemical properties of useful minerals and gangue minerals. Such physicochemical properties are mineral specific gravity, magnetic, electrical, and surface chemical properties, and correspondingly the mineral separation and enrichment methods consist of gravity concentration, magneto-electric beneficiation, flotation, respectively, which are applied in a fairly small area because of the limitations imposed by the difficulties in changing mineral specific gravity, magnetic and electrical properties. However, the artificial change in mineral surface properties can be achieved, thus flotation has been widely used in mineral processing engineering, so to speak, all mineral separation and concentration can be operated by flotation method.

It is universally accepted that chemistry is an important and basic subject for mineral processing engineering disciplines because flotation process, the theoretical basis of which is chemistry, is widely used in mineral separation and enrichment. As a primary mineral processing method, flotation itself is the application of chemistry in mineral processing engineering disciplines. Therefore, chemical education plays a very important role in the theoretical education of mineral processing engineering disciplines.

Minerals and rocks are naturally formed in nature, with a fixed composition of the solid compounds. Mineral surface properties are different due to different compositions and metallogenic conditions, and even the same composition of minerals will have different surface properties because of different mineralization location and metallogenic conditions. With regard to the flotation separation of minerals, there are some similarities in different mineral surface properties. The similari- ties and differences of surface properties between minerals and rocks, minerals and minerals, rocks and rocks serve as a basic principle for flotation separation and enrichment of these minerals. In flotation engineering, the surface properties of minerals and rocks can be artificially adjusted and changed as needed, thereby expanding the difference of the surface properties between minerals separated, to achieve effective separation between the minerals[1].

Chemistry is a basic course that students need to learn from junior high school and it remains to be a compulsory course for the doctoral students in mineral processing engineering. Even the high-level professionals and professors specialized in mineral processing engineering keep studying Chemistry. Since flotation and Chemistry cannot be separated, there is always more Chemistry knowledge to study for flotation. How profound the chemistry is; how esoteric the flotation will be. Chemistry education is one of the foundations in training talents of mineral processing engineering, with different levels of talents corresponding to different levels of chemical education. Only the importance is attached to chemical education, can talent training in mineral processing disciplines be well realized.

\section{Flotation is the Application of Chemistry in Mineral Processing Engineering}

Inorganic chemistry is the most ancient subdiscipline of chemistry, studying elements, simple substance and source, preparation, structure, nature, change and application of inorganic compounds. The object of flotation is mineral rock, the inorganic substance indeed, the mineral surface properties of which are determined by the structure and nature of the mineral itself, thus the study of mineral surface nature can not be separated from the study of the mineral internal composition, structure and nature. The study of minerals and rocks covers all 
areas and contents of inorganic chemistry, making inorganic chemistry a required course for students in mineral processing engineering.

As a remarkably important branch of chemistry, organic chemistry, also known as the chemistry of carbon compounds study the structure, nature and preparation of organic compounds. Carbon compounds are called organic compounds because the previous chemists maintained that carbonaceous material cannot be created but by organisms. However, in 1828, since the German chemist Friedrich Weller, successfully synthesized urea (a kind of biological molecules) in the laboratory, organic chemistry has gone far away from the traditional scope of the definition, expanding as the carbonaceous materialthe chemical. Mineral flotation is achieved by changing the hydrophobicity of the mineral surface, while the increase in mineral surface hydrophobicity is operated by adsorbing on mineral surface with heteropolarity molecules containing alkyl, which are typical organic matter molecules, concerned in the study of flotation agents like collector agent, foaming agent, covering a wide range of organic chemistry. Hence organic chemistry is also a required course for students in mineral processing engineering.

The contents of physical chemistry can be roughly categorized into three aspects: macroscopic equilibrium properties of chemical system, microstructures and properties of chemical system and the dynamic nature of chemical system. Taking three fundamental laws of thermodynamics as the theoretical basis, the first aspect studies the macroscopic chemical system's balanced physicochemical properties and regularity in the gaseous, liquid, solid, dissolved, and the highly dispersed states. The physicochemical subdisciplines including chemical thermodynamics, solution, colloid and surface chemistry belong to this aspect. Theoretically based on quantum theory, the second aspect studies the structure of atoms and molecules, the space structure of atoms and molecules at the bulk phase of objects, the surface structure, as well as the regularity of the structure and physical properties. The physicochemical subdisciplines including structural chemistry and quantum chemistry belong to this aspect. The third aspect studies the velocity of the chemical changes and change mechanism in the system process caused by the perturbation of the chemical or physical factors. The physicochemical subdisciplines including chemical kinetics, catalysis, photochemistry and electrochemistry belong to this aspect.

With rich connotation and broad extension, physical chemistry covers all aspects of flotation such as mineral rock, slurry solution, organic molecules, the interaction between the gas medium in froth flotation with the mineral, etc. Physical chemistry is a critical course in mineral processing engineering disciplines, with the time allotment of most class hours in two semesters set in the undergraduate curriculum. Physical chemistry study is directly related to the flotation study. Physical chemistry is also a compulsory course for the postgraduate entrance examination of mineral processing engineering disciplines.

The main contents of analytical chemistry include the material element, the qualitative analysis of the group, quantitative analysis of the quantity of each ingredient or substance purity; the structure and three-dimensional analysis of spatial arrangement of the molecules formed with matter atoms. The object of study ranges from a single quality to a complex mixture and macromolecular compounds, from inorganic to organic com- pounds, from low molecular weight to high molecular weight. Samples may be gaseous, liquid and solid. Sample weight falls into the scope of 100 grams above to mg or below. Determination of residue proposed by E. Wesson Berg in 1931 specifies that only 10 micrograms of samples belong to the analysis of ultramicro amounts. The instruments used range from the test tube to the advanced instruments attached with automation equipment and computer program control, recording and storage. Analytical chemistry is based on fundamental chemical theory and experimental techniques, absorbing the knowledge of physics, biology, statistics, computer and automation to enrich its content, which aims to solve various problems of scientific and technical analysis. As for mineralized rocks, solution, organic and inorganic agents, mineral processing raw materials and products involved mineral processing engineering disciplines, their qualitative or quantitative description will not be achieved without analyzing and detecting. Instrumental analysis and detection in theory research process is indispensable to study and understand the behavior mechanism of mineral processing. Therefore, Mineral processing is closely related to analytical chemistry. The curriculum of mineral processing engineering disciplines requires systematical study of analytical chemistry at the undergraduate or graduate level.

Being more detailed chemical branch directions than the four chemistries above, structural chemistry, polymer chemistry, complex chemistry, electrochemistry, quantum chemistry, will be involved to varying degrees for specific content and purpose during the study of mineral flotation. In order to make a closer combination of chemical and mineral processing engineering disciplines, the curriculum at the graduate level offers a flotation surface chemistry, the chemistry of the flotation reagents, flotation electrochemistry and flotation solution chemistry.

Although a lot of chemistry courses are set up in different stages of mineral processing engineering disciplines, covering almost all the contents of the field of chemistry, it is still a long way to go with respect to in-depth study and understanding of flotation. Mineral flotation has greatly developed by now, yet a lot of flotation theories remain unsolved and the level of flotation process calls for improvement. To further strengthen chemical education and mineral flotation chemistry research is significantly essential for the development of mineral flotation.

\section{Chemistry Education in Talent Training at All Levels}

In the case of secondary and vocational education aiming to train skilled workers, the students are mostly junior and senior high school graduates with quite limited fundamental chemical knowledge and especially the junior high school students can simply understand the preliminary chemical phenomena. Thus the instruction of mineral processing expertise requires the supplement of chemical knowledge necessary for flotation technology either by integrating it into the learning of expertise or offering an independent basic chemistry course. Students must understand and master the basic chemical knowledge demanded in flotation technology before professional courses of flotation technology is efficiently carried out and students really master the technical knowledge of mineral flotation.

In the case of specialty education and undergraduate education targeting production technology management and application, the curriculum system has considered the importance of the chemistry for mineral processing engineering. Inorganic 
chemistry, organic chemistry and physical chemistry are all required courses, holding a large share of class hours in the basic specialty curriculum. After systematically learning chemical knowledge, students have been able to thoroughly understand chemical problems in mineral flotation, but also better master the theory of flotation and flotation process expertise while learning flotation professional courses, and employ learned chemical knowledge and flotation theory based on the changes in the nature of the ore, to analyze and solve the general technical problems in the production technology management and application process.

In the case of graduate education directing at scientific research, in order to enable students discover and resolve production technical problems, to equip them with capability of independently undertaking scientific research in the field of mineral processing engineering, analytical chemistry is further required to learn on the basis of inorganic chemistry, organic chemistry and physical chemistry at undergraduate education. Learning of analytical chemistry enable graduate students to master the conventional analytical techniques, to master the modern detection methods used in scientific researches, to discover, analyze and study test data, results of researches, finally solving scientific and technical issues. In case of doctoral students, so as to strengthen their knowledge of the mechanism of mineral flotation and cultivate their innovative spirit and awareness, they are expected to study quantum chemistry to explore mineral flotation at the level of electrons, atoms, molecules, after a quite sound grasp of organic chemistry, inorganic chemistry, physical chemistry and analytical chemistry.

Seen from the discussion above, flotation is closely bonded to chemistry as a part of applied chemistry. It is hard to imagine the technique application and development and theoretical research of flotation for both technical workers and flotation-exploring doctoral students without the foundation of chemistry. As a basis for flotation which is the vital method in mineral processing engineering, education of chemistry is extremely significant mineral processing engineering disciplines.

\section{Attach Importance to Chemistry Education in Mineral Processing Engineering Disciplines}

Since mineral resources are unsustainable, resource depletion has become one of the important issues constraining social and economic development with the continuous development and utilization, thus mineral resources development and utilization must adhere to the principle of efficient use. In fact, the development and utilization of advanced technology of mineral processing props up the efficient utilization of resources. Flotation is the crucial method in mineral processing, and it is on chemistry that flotation is based. Recycling and utilization of mineral resources is indeed employing chemical technology or surface chemical technology. Only great emphasis is attached to chemistry education in mineral processing engineering disciplines, can the quality of talent be improved radically and the fundamental problem of efficient utilization of mineral resources be solved from the source.

Out of exploitation, a variety of paragenetic and associated resources in complex nature bring lots of difficulties to their separation and enrichment. In order to realize the comprehensive utilization of resources, any valuable minerals should be recycled. At this time, the technologies, related to chemistry indeed, including the physical and chemical properties of these minerals, chemical change of the various minerals' nature and enlargement of attribute distinction from one another become important research projects of processing and engineering disciplines. Therefore, Chemistry in mineral processing engineering disciplines determines the comprehensive utilization of mineral resources. Accordingly, only great emphasis is attached to chemistry education in mineral processing engineering disciplines, can the quality of talent be improved radically and the fundamental problem of efficient utilization of mineral resources be solved from the source.

Mineral resources were formed naturally, containing components beneficial to human society as well as harmful components. In the process of recovery and use of mineral resources, while efficiently and comprehensively recycling useful components, dealing with the harmful ingredients is one of the tasks in mineral processing engineering disciplines. Only by handling hazardous components and making the useless components remained during and after mineral processing harmless to human and society, can the clean utilization of mineral resources be achieved. The handling of hazardous components necessitates knowledge of these components' nature, and then application of chemical and physical methods for separation and harmless treatment, which are closely related with chemistry, so clean use of mineral resources can not be separated from the chemistry. Chemistry education is required in mineral processing engineering disciplines for clean utilization of mineral resources[2].

Mineral processing mostly involves ore pulverizing, dissociating useful minerals from gangue minerals, which is chiefly conducted in the water, leaving wastewater discharge to be the important issue affecting the environment. Today's mineral processing engineering calls for zero discharge of wastewater to ensure that wastewater does not impact on the environment. Zero discharge of wastewater necessitates wastewater recycling and reuse that will bring problems affecting the beneficiation technical indexes, which should be avoided as much as possible by studying the nature of backwater and even appropriate chemical treatment involving chemical knowledge. Hence wastewater's impact on the environment and wastewater reuse treatment are directly related to chemistry. The environmental problems brought by mineral processing calls for emphasis to chemistry education in mineral processing engineering disciplines[3].

During mineral processing, sustainable development of mineral resources development and utilization can be achieved if we can fulfill efficient, integrated and clean use of resources, wastewater recycling and zero discharge, all of which are closely related to chemistry. Therefore, chemistry is so important in mineral processing engineering that chemistry education becomes an essential requirement in this field.

\section{Conclusion}

(1) The widely used flotation is actually a branch of applied chemistry in mineral processing process. All kinds of chemistry will be involved in various aspects of flotation: study of mineral rock is relevant to inorganic chemistry and crystal chemistry; the interaction between slurry solution and mineral surface and the slurry solution properties are associated with the physical chemistry; mineral collector agent and agentia of foaming agent are related to organic chemistry; the analysis of the properties of minerals and rocks, solution and agents, and product exami- 
nation and testing cannot be done without analytical chemistry. Additionally, the in-depth study of flotation theory and the revealing of the nature of flotation call for further knowledge of quantum chemistry, electrochemistry, structural chemistry, complex chemistry, etc. Chemistry plays an extremely important role in mineral processing engineering disciplines.

(2) The talent training in mineral processing area should be conducted in accordance with students' aptitude and their fundamental knowledge of chemistry. Major courses should be offered, and especially flotation specialized courses are a critical factor to improve the quality of talent cultivation. To train the high-level professionals like graduate students with master and doctor degrees, analytical chemistry and further the quantum chemistry should be included in their courses because the undergraduate courses like organic chemistry, inorganic chemistry and physical chemistry are limited. Their chemical knowledge level lays the ground for the study of mineral processing expertise.

(3) Mineral processing engineering disciplines are directly connected with the use of mineral resources in efficiency, inte- gration and cleanliness and environmental protection, which cannot be achieved without the critical knowledge of chemistry. Improving the chemistry education and expertise of professionals in mineral processing engineering fields is an important guarantee for sustainable development of mineral resources exploitation and utilization.

\section{REFERENCES}

Si-Qing Liu, Shu-Ming Wen, Dian-Wen Liu, Xiong Tong, Zuo-Yue Lan,"Some Thoughts on Educatiing Professionals for Ming Industry," IEMI2012, Volume 3, pp. 1774-1777.

Wen Shuming, Yang Yi, Liu Dianwen, "Alleviation of talent supply and demand contradictions throuth non-academic education strategy in ming engineering," China Education Thecnology and Equipment, vol.15, no.237, pp.8-10. (in Chinese)

Jianfang $\mathrm{Xu}$, "A research of the undergraduates information quality education and innovation ," Journal of Jilin Institute of Chemical Technology, vol.29, no.2, pp.104-106,Feb. 2011. (in Chinese) 